\title{
Estrogen Promotes cAMP Production in Mesenchymal Stem Cells by Regulating ADCY2
}

\author{
Guangfeng Zhao ${ }^{1}$, Xiujun $\mathrm{Li}^{1}$, Huishuang $\mathrm{Miao}^{2}$, Shiwen Chen ${ }^{1}$, Yayi $\mathrm{Hou}^{2}$ \\ ${ }^{1}$ Department of Obstetrics and Gynecology, Nanjing Drum Tower Hospital, Nanjing University Medical School, Nanjing, China \\ ${ }^{2}$ The State Key Laboratory of Pharmaceutical Biotechnology, Division of Immunology, Medical School, Nanjing University, Nanjing, China
}

Background and Objectives: The maternal-fetal interface is an important source of mesenchymal stem cells (MSCs), and it is influenced by high levels of estradiol (E2) during pregnancy. It is highly important to study the role of E2 in MSCs for both clinical application and understanding of the mechanisms underlying pregnancy related diseases. Methods and Results: In this study, differently expressed genes (DEGs) were found in the MSCs after exposure to E2. Then, Gene Ontology (GO) and Kyoto Encyclopedia of Genes and Genomes (KEGG) pathway enrichment analysis of DEGs was performed and the integrated regulatory network of DEGs-miRNA was constructed. A total of 390 DEGs were found in the MSCs exposed to E2, including 164 upregulated DEGs (e.g. ADCY2, VEGFA and PPY) and 226 downregulated DEGs (e.g. KNG1, AGT and NPY). Additionally, 10 miRNAs (such as miR-148A/B, miR-152, miR-182) identified the integrated regulatory network of DEGs-miRNAs. Among them, the expression of ADCY2 was significantly upregulated, and this was associated with multiple changed genes. We confirmed that the expression of ADCY2 is significantly promoted by E2 and subsequently promoted the production of cAMP in MSCs. We also found that E2 promoted ADCY2 expression by inhibiting miR-152 and miR-148a.

Conclusions: E2 promotes the expression of cAMP through miR-148a/152-ADCY2 in MSCs. It is suggested that E2 plays a key role in the growth and function of MSCs.

Keywords: Estradiol, Mesenchymal stem cells, miRNAs, ADCY2, cAMP

Received: November 15, 2019, Revised: January 25, 2020, Accepted: January 30, 2020, Published online: February 29, 2020 Correspondence to Guangfeng Zhao

Department of Obstetrics and Gynecology, Nanjing Drum Tower Hospital, Nanjing University Medical School, 321 Zhongshan Rd., Nanjing 210008, China

Tel: +86-25-83106666, Fax: +86-25-83106666

E-mail: guangfeng_zhao@126.com

Co-Correspondence to Yayi Hou

The State Key Laboratory of Pharmaceutical Biotechnology, Division of Immunology, Medical School, Nanjing University, 21 Hankou Rd., Nanjing 210093, China

Tel: +86-25-83686441, Fax: +86-25-83686441

E-mail: yayihou@nju.edu.cn

(c) This is an open-access article distributed under the terms of the Creative Commons Attribution Non-Commercial License (http://creativecommons.org/ licenses/by-nc/4.0/), which permits unrestricted non-commercial use, distribution, and reproduction in any medium, provided the original work is properly cited.

Copyright (c) 2020 by the Korean Society for Stem Cell Research

\section{Introduction}

Mesenchymal stem cells (MSCs) are pluripotent stem cells that can differentiate into a variety of cell types, such as chondroblasts, osteoblasts, and adipocytes and they are easily cultured and stored in vitro $(1,2)$. MSCs have always been a hot topic in the research world, whether in basic research or in clinical translational research $(3,4)$. Currently, more than 600 clinical trials that used MSCs to treat different diseases are being carried out worldwide, due to the immunosuppressive and angiogenic functions of MSCs (http//www.clinical-trials-com).

The maternal-fetal interface is one of the main sources of $\operatorname{MSCs}(5,6)$, which maintain maternal fetal immunity and promote angiogenesis. Specifically, estrogen levels change significantly during pregnancy. At this time, estrogen is produced mainly by the placenta through the conversion 
of androgen precursors. This leads to a significantly higher level of estrogen in the peripheral blood of pregnant women than in that of non-pregnant women (7).

However, the serum levels of E2 were found to be significantly lower in women with preeclampsia (PE) than among controls $(8,9)$. In accordance with this, the MSCs of the maternal fetal interface in PE patients, whether they come from the decidua or the umbilical cord, show slow growth, senescence, and impaired immunosuppression $(10,11)$. After treatment with normal MSCs, the PE symptoms of PE-like mice were alleviated significantly (12). These findings suggest that E2 may play an important role in promoting placental MSCs. In this way studying the role of E2 in MSCs is of great significance because of both clinical application and understanding of the mechanisms underlying pregnancy related diseases.

In this study, we aimed to explore the regulatory mechanisms underlying E2 in MSCs. The gene expression profile was analyzed after $24 \mathrm{~h}$ of treatment with E2. We then performed the Gene Ontology (GO) and Kyoto Encyclopedia of Genes and Genomes (KEGG) pathway enrichment analysis of DEGs. We also constructed an integrated regulatory network of DEGs-miRNA. Through bioinformatics analysis and in vitro experiments, we found that E2 promotes the expression of ADCY2 through miR-148a/152, thereby promoting cAMP synthesis in MSCs.

\section{Materials and Methods}

\section{MSC isolation, culture and E2 treatment}

MSCs were isolated from fresh umbilical cords within $4 \mathrm{~h}$. The umbilical cords were obtained from the normal pregnant women undergoing cesarean section in Obstetrics and Gynecology Department of Nanjing Drum Tower Hospital. This study was reviewed and approved by the Ethics Committee of Drum Tower Hospital, Nanjing University Medical School and our research was carried out in accordance with the Helsinki Declaration. Written informed consent was obtained from all participants. The MSC separation method is based on our previous report (2). Briefly, the umbilical cord was first rinsed with PBS containing penicillin and streptomycin. The arteries and veins in the umbilical cord were then stripped and the remaining umbilical cord tissue was cut to pieces. Then, the tissues were digested at $37^{\circ} \mathrm{C}$ for $3 \mathrm{~h}$ with enzymes. After termination of digestion with DF12 medium containing FBS, the digestive mixture was filtrated through 40 um screen mesh. Subsequently, the supernatant was centrifuged and suspended with DF12 complete medium. Then the cells were incubated at $37^{\circ} \mathrm{C}$ in a $5 \% \mathrm{CO}_{2}$ saturated humidified at- mosphere. When the cells were spread to $80 \%$, they were digested and passaged with $0.25 \%$ trypsin. MSCs were characterized by flow cytometry for the expression of CD11b, CD73, CD90, CD29, CD44, CD45, CD106, HLA-DR, CD19, and CD14. MSCs were treated with E2 (100 nM) for 24 $h$ as the E2 group; and the cells were treated with the same amount of medium for $24 \mathrm{~h}$ as the control group.

\section{Quantitative real-time PCR}

The methods of total RNA isolation and quantitative real-time PCR were described in our previous study (13). RNA was isolated according to a standard Trizol reagent kit (Invitrogen) protocol. cDNA was synthesized through reverse transcription according to a standard reverse transcription kit (Takara, Japan) protocol. Quantitative realtime PCR was performed on LC480 (Roche). All reactions were run in duplicate. GAPDH served as an internal reference gene and all qPCR data were normalized to GAPDH.

\section{RNA extraction and purification}

Trizol reagent (Cat\#15596-018, CA, USA) was used to extract total RNA according to the manufacturer's instructions. RNA Integrity Number (RIN, 1 to 10) based on the ratio of the $18 \mathrm{~S}$ to $28 \mathrm{~S}$ ribosomal subunits could inspect RNA integration by an Agilent Bioanalyzer 2100 (Santa Clara, CA, USA). RNA with RIN (>7.0) and 28S/18S ( $>0.7$ ) is up to standard. Then RNeasy Mini Kit (Cat \#74106, Qiagen, Germany) and RNase-Free DNase Set (Cat \#254, Qiagen) were used for further purification.

\section{RNA amplification and labeling}

The purified RNA was amplified and labeled by Low Input Quick Amp Labeling Kit, One-Color (Cat\#5190-2305, Agilent) according to the manufacturer's instructions. Then labeled cRNA was purified using the RNeasy mini kit based on the RNA purification.

\section{Hybridization of slides}

A gene expression hybridization kit (Cat\#5188-5242, Agilent) was used for hybridization of slides (Probe name version: Agilent-014850 Whole Human Genome Microarray $4 \mathrm{x} 44 \mathrm{~K}$ ) with $1.65 \mu \mathrm{g}$ of Cy3-labeled cRNA at $65^{\circ} \mathrm{C}$ and $10 \mathrm{rpm}$ for $17 \mathrm{~h}$ in a hybridization oven (Cat\#G2545A, Agilent) according to the manufacturer's instructions. Then slides were washed with a gene expression wash buffer kit (Cat\#5188-5327, Agilent).

\section{Data normalization}

An Agilent microarray scanner (Cat\#G2565CA) was used to scan slides. Subsequently, data were extracted using 
Feature Extraction software 10.7 (Agilent). Raw data were normalized using the Quantile algorithm with Gene Spring Software 11.0 (Agilent). Lastly, eight samples were obtained including four E2 samples (E2-1, E2-2, E2-3, E2-4) and four control samples (Ctl-1, Ctl-2, Ctl-3, Ctl-4).

\section{Identification of DEGs}

The limma package (14) in R software was used to identify the DEGs between the E2 and control groups according to the expression values of the probe in the slides. The threshold value of DEGs was set as $\mid \log$ fold change (FC) $\mid>1$ and $p$ value $<0.05$. Then, the clustering heatmap was constructed using the pheatmap function in $\mathrm{R}$ package (version 1.0.8, https://CRAN.R-project.org/package=pheatmap).

\section{Function and pathway analysis of the DEGs}

The GO and KEGG pathway enrichment analysis of DEGs was used to assess the Molecular Function (MF), Biological Process (BP), Cellular Component (CC) and pathway of DEGs via DAVID 6.8. $\mathrm{p}$ value $<0.05$ was significantly different.

\section{Analysis of PPI}

PPI network was constructed with DEGs using STRING (Version 10.0, http://www.string-db.org/) under a threshold of combined score $>0.4$ (required confidence), and was visualized by Cytoscape software (Version 3.4.0, http://cytoscape.org/). In addition, CytoNCA (Version 2.1.6, http://apps.cytoscape.org/apps/cytonca) was used to analyze the network topology, and the parameter was set as "without weight" (15). The importance of each node within the network was analyzed according to degree centrality (16), betweenness centrality (17) and closeness centrality (18). In the PPI network, proteins with similar function were clustered together, and the distance between nodes was related to their functions. In this way, MCODE module analysis could predict the unknown functions of proteins in the network (19).

\section{Integrated regulatory network of DEGs-miRNA}

Webgestalt was used to identify the miRNA based on DEGs. The miRNAs with the count of DEGs $\geq 5$ and the adjust $\mathrm{p}$ value $<0.001$ were identified. The integrated regulatory network of DEGs-miRNA was constructed based on the PPI network, and miRNA-regulatory networks.

\section{Western blot analysis}

MSCs cells were washed two times with PBS and suspended with ice-cold lysis buffer supplemented with $1 \%$ PMSF (protease inhibitor) incubated on ice for $30 \mathrm{~min}$ - utes. The total protein concentration was tested by a BCA Protein Assay Kit. Subsequently, loading buffer was added to each sample and boiled at $100^{\circ} \mathrm{C}$ for $10 \sim 15$ minutes. Protein samples $(30 \mu \mathrm{g})$ were resolved by $10 \%$ SDS-PAGE and transferred to poly vinylidene difluoride membranes at $100 \mathrm{~V}$ for $1 \mathrm{~h}$. The membranes were blocked using $5 \%$ skimmed milk in TBST for 2 hours at room temperature and subsequently incubated overnight at $4^{\circ} \mathrm{C}$ with diluted primary antibodies against ADCY2 (Santa Cruz, sc-514938) and $\beta$-actin (Cell Signaling Technology, \#3700), followed by incubation with the appropriate horseradish peroxidase-conjugated secondary antibody (Cell Signaling Technology) at room temperature for $40 \sim 60$ minutes. The immunoreactive blots were visualized using an ECL-PLUS Kit according to the manufacturer's instructions. The relative protein expression levels were normalized to that of $\beta$-actin.

\section{cAMP assay}

cAMP levels in culture supernatants from E2 treated MSCs were quantified by an enzyme-linked immunosorbent assay (ELISA) kits (Elabscience, China) according to the manufacturer's instructions. All assays were duplicated.

\section{RNAi transfection}

The small interfering RNA specific to ADCY2 (siADCY2) was transfected by Lipofectamine 3000 reagent (Invitrogen, USA) according to the manufacturer's instructions. Briefly, when the MSCs reached about 50\% confluence in 24-wells plate, they were transfected with siADCY2 $(100 \mathrm{nM})$ and negative control siRNA $(100 \mathrm{nM})$ in serum-free medium using Lipofectamine 3000 . After incubation for $4 \mathrm{~h}$ at $37^{\circ} \mathrm{C}$, 400 ul DF12 complete medium was added.

\section{Luciferase activity assay}

The luciferase reporter plasmid containing the 3'UTRs of ADCY2 was constructed by GENEray (GENEray, Biotechnology, Shanghai). MSCs were co-transfected with $50 \mathrm{nM}$ miR-148a or miR-152 mimics or negative control and $200 \mathrm{ng}$ luciferase reporter plasmid in each well. After 4 hours incubation, the cells were maintained in DF12 complete medium for 24 hours and used for luciferase reporter assay. The Dual-Luciferase Reporter Assay was performed following the kit's instruction (Promega, Mannheim, Germany) using GloMax 96 Microplate Luminometer (Promega, Mannheim, Germany).

\section{Statistical analysis}

The experiments were performed at least in triplicate, and all data are expressed as mean \pm S.E.M. All data were 
analyzed using Graphpad prism version 5.01. Differences between two groups were analyzed by a two-tailed Student's t-test. Multiple comparisons were assessed by ANOVA. $\mathrm{p}$ values $<0.05$ were considered significant.

\section{Results}

Changes in the transcriptome in MSCs exposed to E2

First, we determined whether E2 changed the phenotype of MSCs. The expression of CD11b, CD106, CD45, CD19, CD14, HLA-DR, CD44, CD73, CD29 and CD90 was analyzed after $24 \mathrm{~h}$ of treatment with $100 \mathrm{nM} \mathrm{E} 2$. The results
A

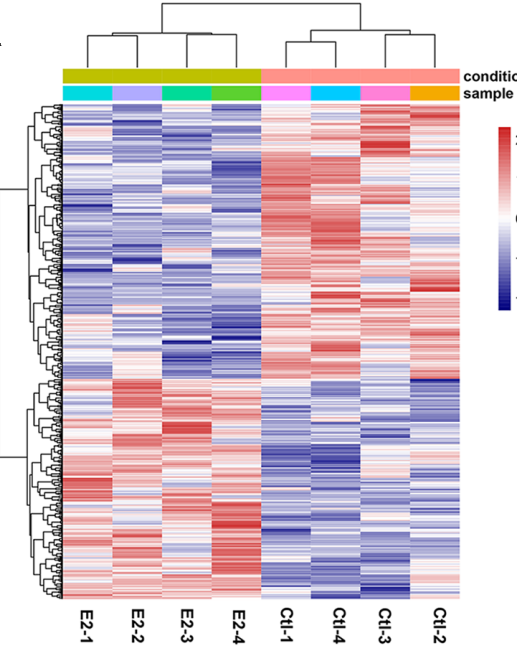

B

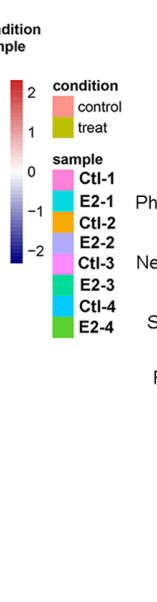

Term

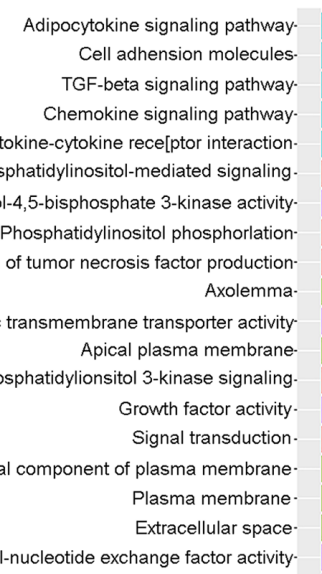

Protein tyrosine kinase

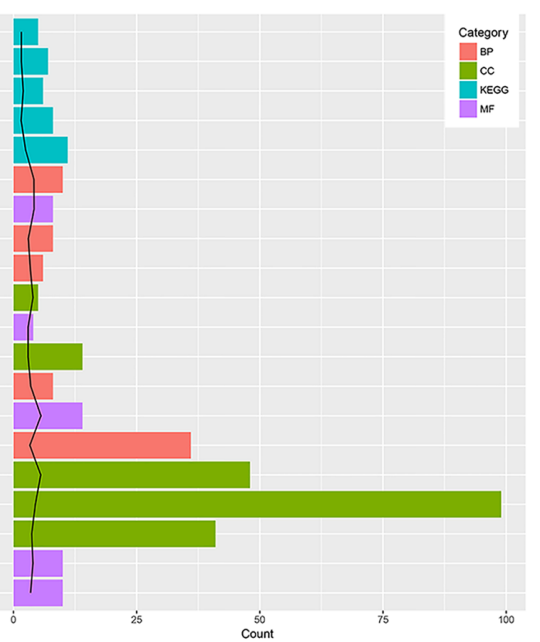

C

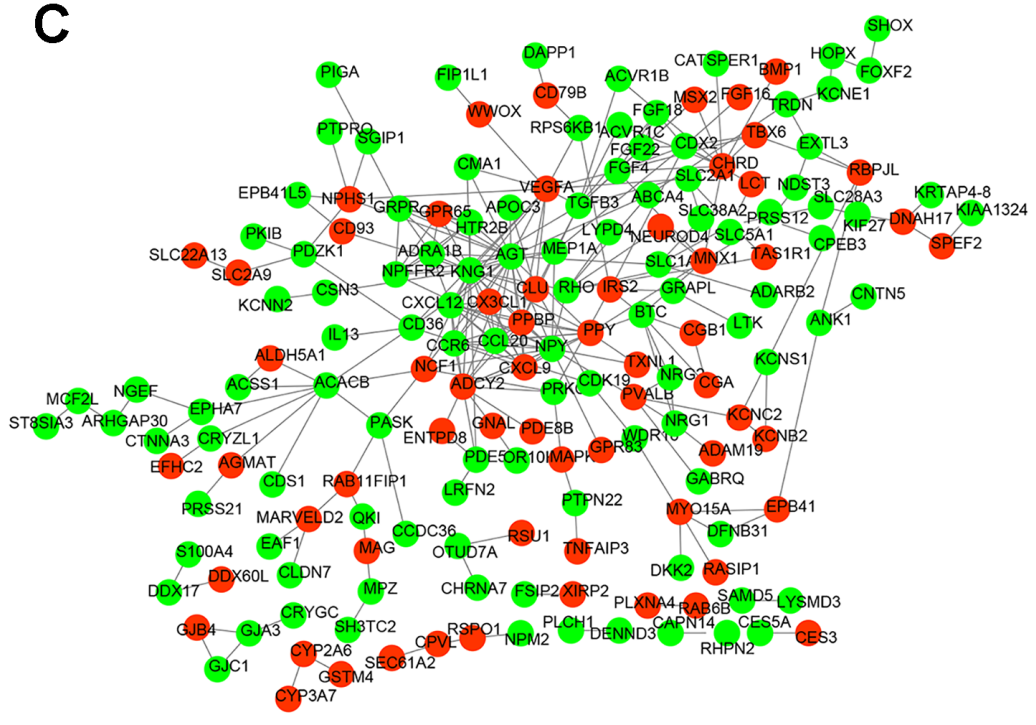

D

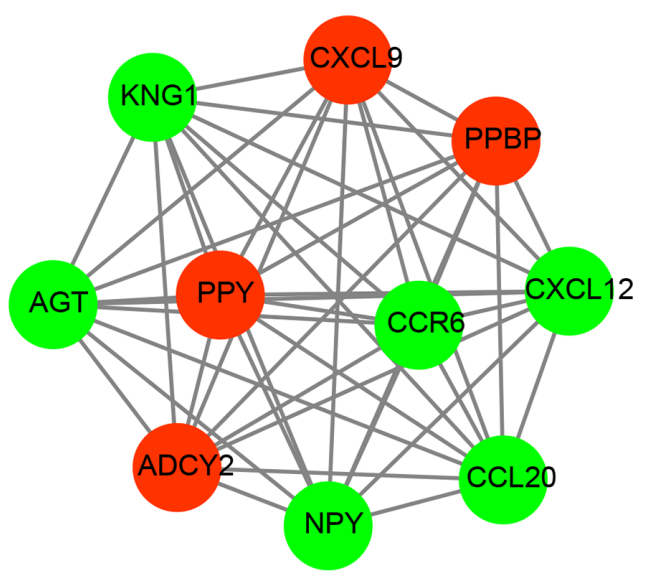

Fig. 1. Changes in the transcriptome of MSCs exposed to E2. (A) The heatmap showed all significantly differentially expressed genes (DEGs) $(p<0.05)$ identified between the MSC treated with E2 and that without E2. The colors blue, white and red indicate increasing levels of DEGs expression. E2-1, E2-2, E2-3 and E2-4 show E2 samples and Ctl-1, Ctl-2, Ctl-3 and Ctl-4 show four control samples. (B) The enrichment results of GO and KEGG pathway (top five $\mathrm{p}$ values). Category: molecular function (MF), biological process (BP), cellular component (CC). Count represents the number of DEGs enriched to the KEGG pathway. Term represents the information of GO function. (C) The protein-protein interaction (PPI) network of DEGs and modules. PPI network, Red nodes represent the up-regulated DEGs; green nodes indicated down-regulated DEGs. The edges between them represent the protein-protein interaction. (D) Modules in PPI, red nodes represent the up-regulated DEGs; green nodes indicate down-regulated DEGs. 
showed that these antigens on the surface of MSCs were not changed after treatment with E2 (Supplementary Fig. S1). Then the DEGs in the MSCs exposed to E2 were analyzed and 390 DEGs were identified, including 164 upregulated DEGs (e.g. ADCY2, VEGFA and PPY) and 226 downregulated DEGs (e.g. KNG1, AGT and NPY). The clustering heatmap is shown in Fig. 1A. These DEGs could be well distinguished between the MSCs treated with E2 and that without E2.

Next, the GO terms of these DEGs were enriched. As shown in Fig. 1B, these DEGs were enriched to cytokinecytokine receptor interaction, TGF-beta signaling pathway, chemokine signaling pathway, and other pathways. Additionally, these DEGs were mainly enriched to signal transduction, phosphatidylinositol-mediated signaling after BP term analysis; plasma membrane, integral component of plasma membrane, and extracellular space after CC term analysis; growth factor activity, Ras guanyl-nucleotide

Table 1. The top 15 of differently expressed genes (DEGs) with higher degree in the protein-protein interaction network

\begin{tabular}{lcllll}
\hline Gene & Degree & Gene & Betweenness & Gene & Closeness \\
\hline KNG1 & 24 & AGT & 4719.9487 & AGT & 0.0316065 \\
AGT & 23 & ACACB & 4226.533 & KNG1 & 0.0315534 \\
NPY & 16 & NPY & 3856.8093 & NPY & 0.031477 \\
ADCY2 & 14 & KNG1 & 3187.0576 & VEGFA & 0.0314243 \\
VEGFA & 13 & VEGFA & 2847.3284 & PPBP & 0.031366 \\
PPY & 12 & CD36 & 2514.4307 & PPY & 0.0313311 \\
PPBP & 12 & PASK & 2474.8 & CD36 & 0.0312963 \\
CXCL12 & 12 & RAB11FIP1 & 1886 & CXCL12 & 0.0312789 \\
TGFB3 & 10 & NCF1 & 1874.909 & ADCY2 & 0.0312731 \\
CXCL9 & 10 & SLC5A1 & 1721.7711 & RHO & 0.0312211 \\
CCR6 & 10 & ADCY2 & 1692.7184 & MEP1A & 0.0312154 \\
CCL20 & 10 & CDX2 & 1498.8306 & CXCL9 & 0.0312038 \\
CHRD & 10 & ABCA4 & 1375.159 & CCR6 & 0.0312038 \\
CDX2 & 9 & EPHA7 & 1358 & CCL20 & 0.0312038 \\
ACACB & 9 & SLC28A3 & 1350 & NCF1 & 0.0311866 \\
\hline
\end{tabular}

exchange factor activity, and protein tyrosine kinase activity after MF term analysis. We also constructed the PPI network and module. There were 170 nodes (individual proteins) and 278 interconnected partners in the PPI network (Fig. 1C). The hub proteins with higher degrees were highly interconnected with other proteins in the PPI network, such as KNG1 (degree: 24), AGT (degree: 23), NPY (degree: 16) and ADCY2 (degree: 14) (Table 1). In the module (score $=10)$, there were 10 nodes and 45 interconnected partners (Fig. 1D).

\section{miRNAs of DEGs in the MSCs exposed to E2}

A total of 10 miRNAs were identified in the miRNA regulatory network (Table 2): miR-148A, miR-152, miR-148B, miR-27A, miR-27B, miR-182, miR-145, miR-153, miR-34B and miR-511. The miRNA-gene network is shown in Fig. 2.

\section{cAMP production, and upregulation of ADCY2 in E2 treated MSCs}

The results of the gene expression profile analysis given above showed that the level of adenylyl cyclase type 2 (ADCY2) had changed significantly. Our in vitro experiments also showed that E2 increased the mRNA and protein levels of ADCY2 in MSCs (Fig. 3A and 3B). ADCY2 is an enzyme that catalyzes the formation of the secondary messenger cyclic adenosine monophosphate (cAMP) from ATP. We further examined the effect of E2 on the level of cAMP in MSCs. We assessed the concentration of cAMP in the culture medium of MSCs that had been treated with $100 \mathrm{nM} \mathrm{E} 2$ for $48 \mathrm{~h}$. As shown in Fig. 3C, E2 promotes the production of cAMP in MSCs. To analyze whether $\mathrm{ADCY} 2$ mediates the promotion of E2 on cAMP, we transfected the small interfering fragment of ADCY2 (siADCY2) into MSCs before E2 treatment and then checked the level of cAMP. The results showed that the concentration of cAMP decreased significantly in siADCY2 pretreated MSCs (Fig. 3D).

Table 2. The miRNAs of DEGs in the MSC exposed to E2

\begin{tabular}{|c|c|c|c|}
\hline miRNA & $\begin{array}{l}\text { Target gene } \\
\text { number }\end{array}$ & $\begin{array}{l}\text { Adjust } \\
\mathrm{p} \text { value }\end{array}$ & Target genes \\
\hline hsa_miR-27A, miR-27B & 11 & $7.56 \mathrm{E}-05$ & $\begin{array}{l}\text { DDK2, ADAM19, ANK1, QKI, RAB11FIP1, CHRD, RPS6KB1, CPEB3, } \\
\text { SLC1A2, CDS1, EPB41 }\end{array}$ \\
\hline hsa_miR-182 & 9 & $8.74 \mathrm{E}-05$ & TXNL1, PLCH1, FOXF2, RAB6B, ADCY2, QKI, PIGA, EPHA7, SLC1A2 \\
\hline hsa_miR-145 & 7 & $0.44 \mathrm{E}-03$ & EPB41L5, ADAM19, RSPO1, ACVR1B, FSIP2, SLC1A2, QKI \\
\hline hsa_miR-148A, miR-152, miR-148B & 7 & $0.80 \mathrm{E}-03$ & CHRD, CDK19, ST8SIA3, ADCY2, SLC1A2, QKI, BMP1 \\
\hline hsa_miR-153 & 6 & $0.80 \mathrm{E}-03$ & RPS6KB1, MAPK4, ADAM19, ANK1, SLC1A2, QKI \\
\hline hsa_miR-34B & 6 & $0.80 \mathrm{E}-03$ & CPEB3, RPS6KB1, NRG1, MAPK4, VEGFA, ADCY2 \\
\hline hsa_miR-511 & 6 & $0.07 \mathrm{E}-03$ & TXNL1, RPS6KB1, KCNE1, FIP1L1, QKI, CDK19 \\
\hline
\end{tabular}




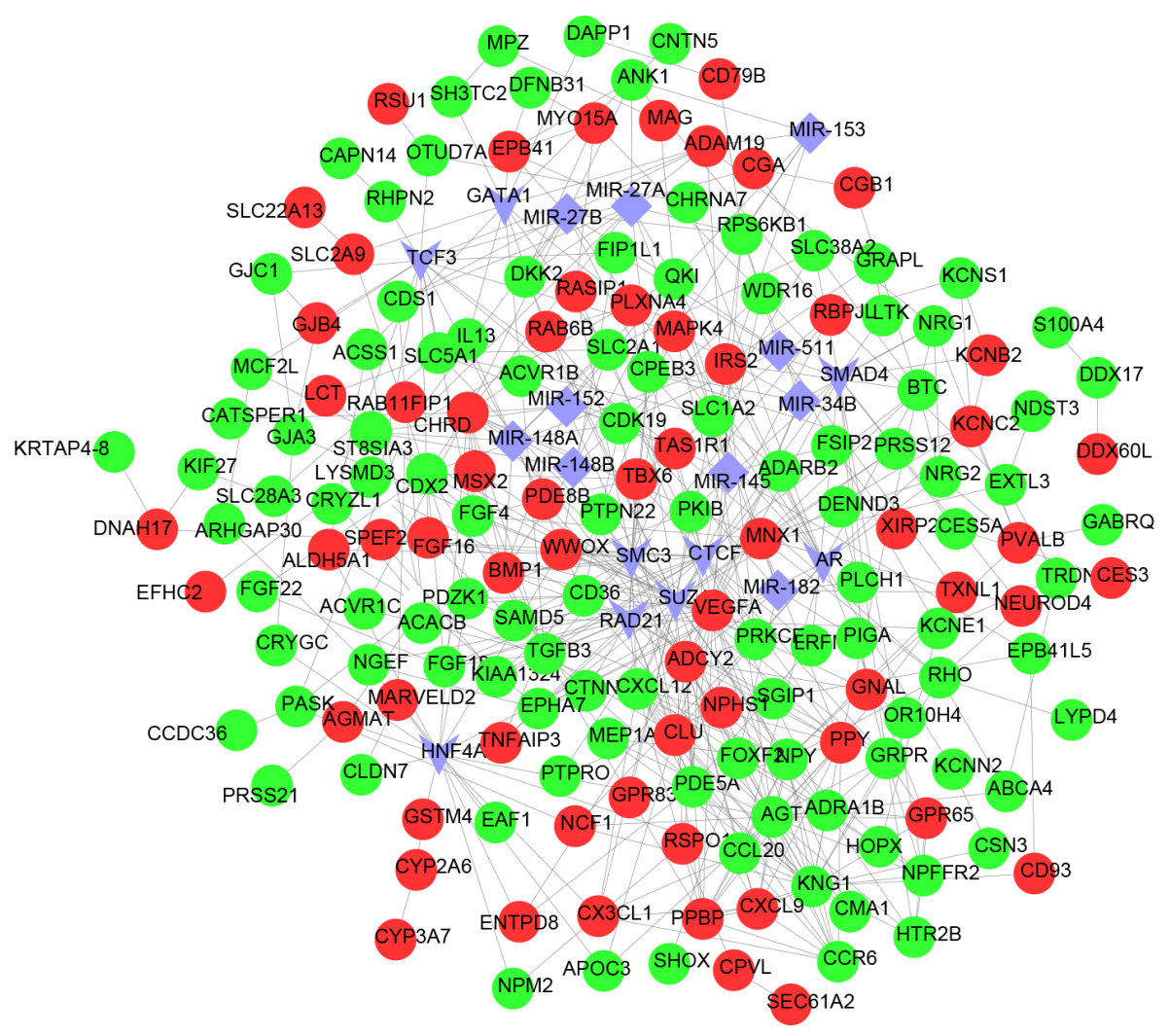

Fig. 2. Integrated regulatory network. Red nodes are up-regulated DEGs, green nodes are down-regulated DEGs, and lines between DEGs represent connections; the purple V-types indicate transcription factors (TFs). The purple rhombuses represent miRNA.
A

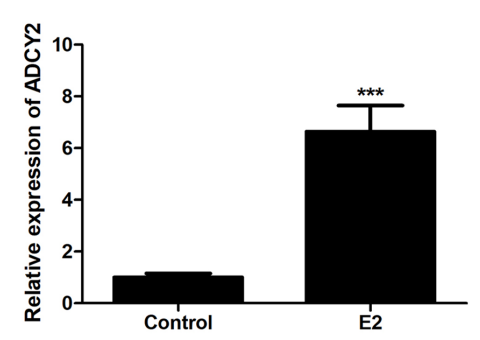

C

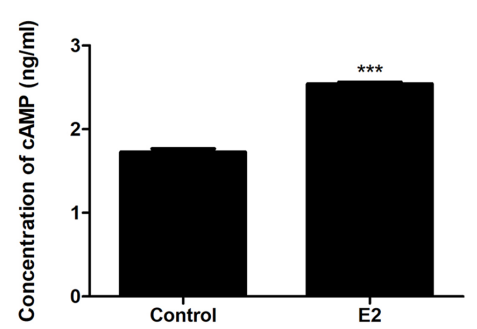

B

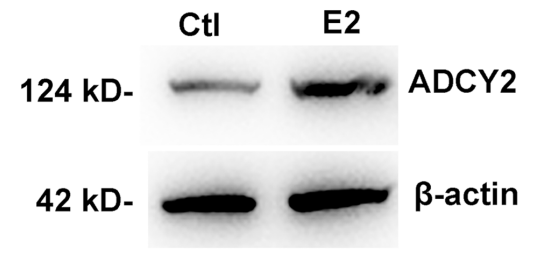

D

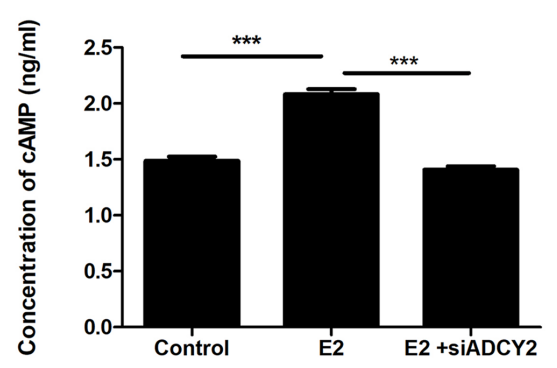

Fig. 3. E2 promotes CAMP production through upregulating $A D C Y 2$ in MSCs. (A) After treatment with 100 nM E2 for $24 \mathrm{~h}$, mRNA level of ADCY2 was assessed using qPCR. (B) After treatment with $100 \mathrm{nM}$ E2 for $24 \mathrm{~h}$, protein level of ADCY2 was detected by western blot analysis. (C) After treatment with $100 \mathrm{nM} \mathrm{E2}$ for $48 \mathrm{~h}$, the concentration of cAMP in the culture medium of MSCs was detected by ELISA. (D) Small interfering fragment of ADCY2 (siADCY2) was transfected into MSCs before E2 treatment. Then the level of cAMP was checked by ELISA. ${ }^{* * *} \mathrm{p}<0.001$.

\section{E2 promotes ADCY2 expression through inhibiting $\mathrm{miR}-148 \mathrm{a} / \mathrm{miR}-152$}

Through bioinformatics analysis, we found a regulatory relationship between the differentially expressed genes and microRNAs after E2 treatment. miRNA has been widely reported to be involved in E2 regulation (20-22). Through miRNA-target gene prediction, we found miR-148a and miR-152 have binding sequences on the 3'UTR of ADCY2 (Fig. 4A). To determine whether miR-148a/miR-152 could regulate $\mathrm{ADCY} 2$ expression, we constructed a luciferase 
A

3' uguuuCAAGAC AUC ACGUGACu 5' miR-148a |||||||||||||| 172: 5' agaucGUUCUGCCACUUGCACUGu 3' ADCY2

3' gguuCAagacAgu ACGUGACu 5' miR-152 |||||||| $\mid$ |||||||

173: 5' gaucGUUCUGCCACUUGCACUGu 3' ADCY2

C

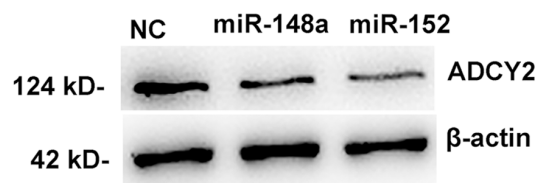

E

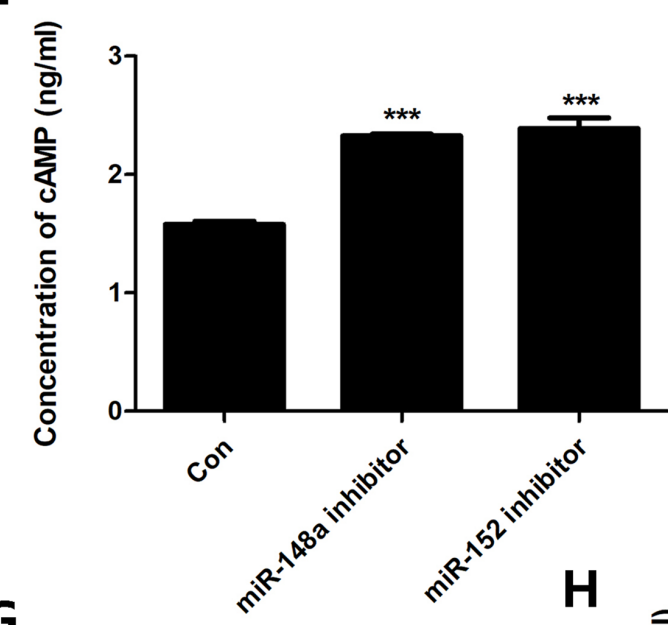

$\mathbf{G}$

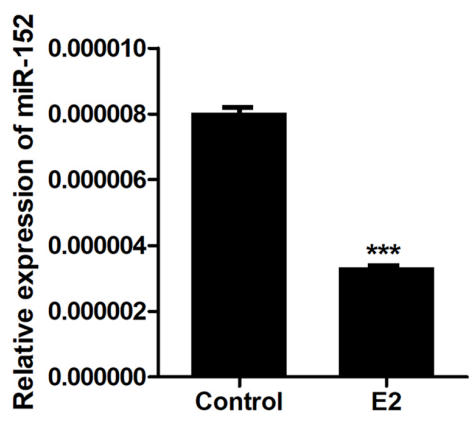

H
B

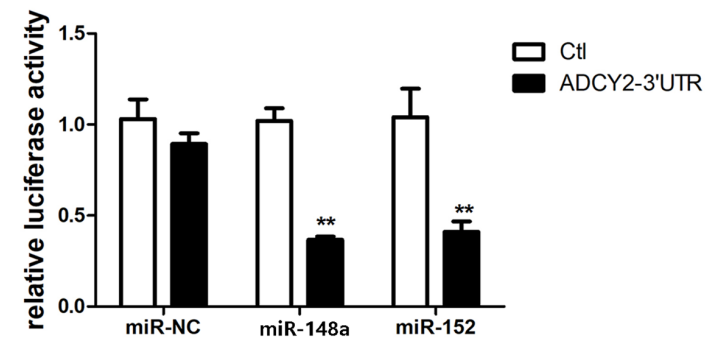

D miR148a miR-152 Ctl inhibitor inhibitor

124 kD-

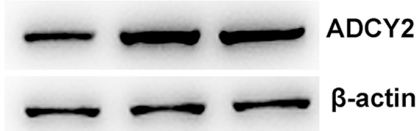

$\mathbf{F}$
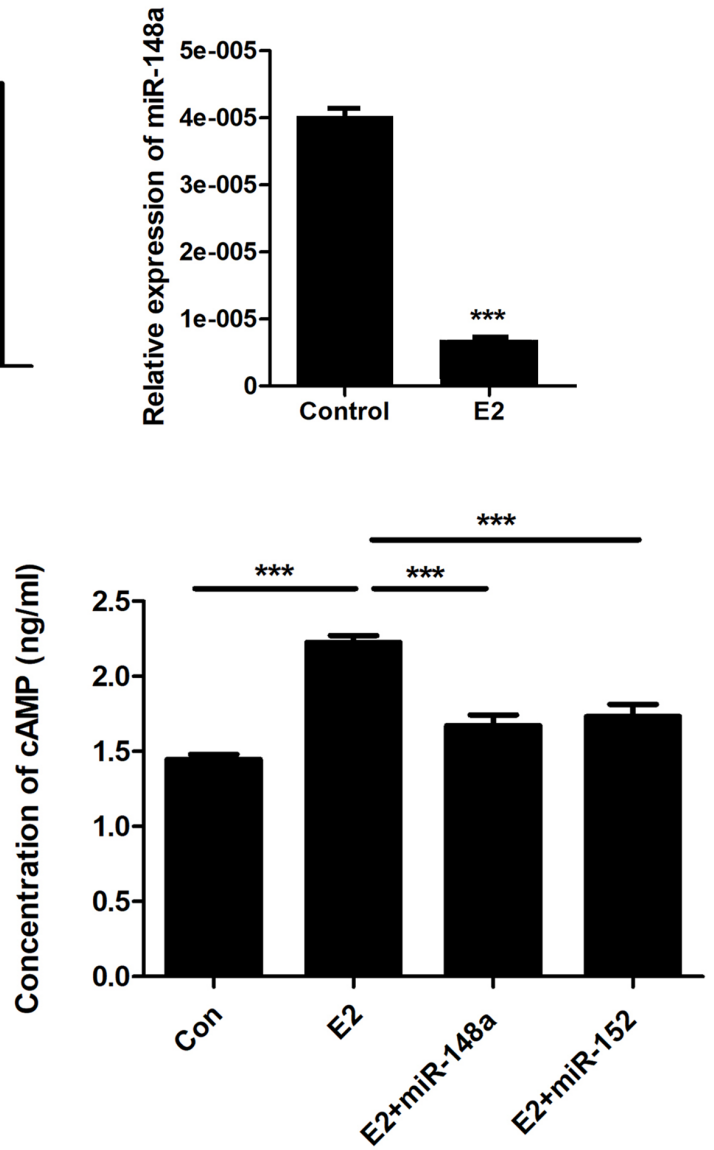

Fig. 4. $E 2$ promotes $A D C Y 2$ expression through inhibiting miR-148a/miR-152. (A) miR-148a and miR-152 have binding sequences on $3^{\prime} U T R$ of ADCY2. (B) Co-transfected with luciferase report plasmid including 3'UTR of ADCY2 and miR-148a/152 into MSCs, then the luciferase activity was detected. (C) After transfection with miR-148a and miR-152 mimics for 48 h, protein level of ADCY2 in MSCs was detected by western blot analysis. (D, E) After transfection with miR-148a and miR-152 inhibitors for $48 \mathrm{~h}$, protein levels of ADCY2 and cAMP in MSCs were detected. $(F, G)$ After treatment with $100 \mathrm{nM} \mathrm{M} \mathrm{E2} \mathrm{for} 24 \mathrm{~h}$, the levels of miR-148a and miR-152 were determined using qPCR. $(\mathrm{H})$ miR-148a or miR-152 mimics were transfected into E2-treated MSCs, and then the expression of cAMP was detected determined by ELISA. ${ }^{* *} \mathrm{p}<0.01, * * * \mathrm{p}<0.001$. 
report plasmid including 3'UTR of ADCY2 and co-transfected it with miR-148a or miR-152 into MSCs. The results showed that both miR-148a and miR-152 inhibited the luciferase activity (Fig. 4B). Both miR-148a and miR152 mimics were found to inhibit the protein levels of ADCY2 (Fig. 4C). On the contrary, after blocking miR148 a or miR-152 using miR-148a or miR-152 inhibitors, the expression of ADCY2 and cAMP was increased in MSCs (Fig. 4D and 4E). Furthermore, we analyzed the effects of E2 on the expression of miR-148a and miR-152 in MSCs. As shown in Fig. 4F and 4G, 100 nM E2 significantly inhibited the expression of miR-148a and miR-152. To determine whether miR-148a/miR-152 mediates the promotion of E2 on the expression of cAMP, we transfected miR-148a or miR-152 mimics into E2-treated MSCs, and then assessed the expression of cAMP. These results showed that miR-148a or miR-152 mimics reversed E2-induced upregulation of cAMP (Fig. $4 \mathrm{H}$ ).

\section{Discussion}

MSCs from placentas and umbilical cords cannot avoid the influence of E2. Therefore, studying the effect of E2 on MSCs will be of great value in the clinical application of MSCs and assessing the pathogenesis of pregnancy related diseases. In this study, we found that E2-induced ADCY2 plays a key role in the growth of MSCs by analyzing gene expression profiles of MSCs. Further analysis and experimental verification revealed that E2 promotes the expression of ADCY2 through microRNA-148a and microRNA-152, thus inducing the synthesis of cAMP in MSCs.

ADCY2 encodes the adenylate cyclase, which can catalyze ATP to form the second messenger cyclic adenosine monophosphate (cAMP) (23). For example, it is reported that ADCY2 is stimulated by activation of Gq-coupled muscarinic receptors to generate localized cAMP through protein kinase $\mathrm{C}$ (PKC) in human embryonic kidney cells (24). cAMP is an important second messenger in cell proliferation, differentiation, apoptosis, inflammation and other biological processes (25) and works by activating protein kinase A (PKA) and further phosphorylation of cAMP response element binding protein (CREB) $(26,27)$. Of note, cAMP also plays an important role in the growth and differentiation of MSCs $(28,29)$. It is even necessary to add cAMP to maintain the growth of MSCs derived from human umbilical cord (30). However, the effect of E2 on the expression of ADCY2 and production of cAMP has only rarely been reported. Through bioinformatics analysis, we found that E2-regulated microRNAs (miRNAs) may affect the expression of ADCY2.

miRNAs are small non-coding RNAs of approximately 22 nucleotides. They are involved in the biological processes underlying human diseases by regulating their target genes. At present, many studies have reported that miRNAs have a key regulatory role in MSCs. Here we predicted that ADCY2 was target gene of miR-152, miR-148b, miR$148 \mathrm{a}, \mathrm{miR}-34 \mathrm{~b}$, and miR-182 in the integrated regulatory network of DEGs-miRNAs. It has been reported that miR$148 \mathrm{a} / 152$ was upregulated in the placenta of preeclamptic rats (31). The expression of miR-152 is increased in the placental tissues and serum of $\mathrm{PE}$, and this increased miR-152 expression can promote the apoptosis of trophoblast cells $(32,33)$. Furthermore, miR-148a could modulate differentiation of MSCs (34), and the expression and function of miR-148a /152 were associated with estrogen signaling pathway (35-38). All these findings suggest that miR-148a and miR-152 are key molecules mediating the function of E2 in MSCs.

In conclusion, we first systematically analyzed the mechanism of E2 on MSCs, and found the key molecular pathway of E2 affecting MSCs. Our findings provide a new molecular target for assessing the pathogenesis of pregnancy related diseases and may promote the clinical application of MSCs.

\section{Acknowledgments}

This work was supported by the Excellent Youth Natural Science Foundation of Jiangsu Province (BK20170051), Jiangsu Province's Key Provincial Talents Program (ZDRCA2016067), the Strategic Priority Research Program of the Chinese Academy of Sciences (XDA16040101) and Six Talent Peaks Project in Jiangsu Province (2016WSW-063).

\section{Potential Conflict of Interest}

The authors have no conflicting financial interest.

\section{Supplementary Materials}

Supplementary data including one figure can be found with this article online at http://pdf.medrang.co.kr/paper/ pdf/IJSC/IJSC-13-s19139.pdf.

\section{References}

1. Karahuseyinoglu S, Cinar O, Kilic E, Kara F, Akay GG, Demiralp DO, Tukun A, Uckan D, Can A. Biology of stem cells in human umbilical cord stroma: in situ and in vitro surveys. Stem Cells 2007;25:319-331 
2. Liu L, Zhao X, Li P, Zhao G, Wang Y, Hu Y, Hou Y. A novel way to isolate MSCs from umbilical cords. Eur J Immunol 2012;42:2190-2193

3. Bianco P, Cao X, Frenette PS, Mao JJ, Robey PG, Simmons PJ, Wang CY. The meaning, the sense and the significance: translating the science of mesenchymal stem cells into medicine. Nat Med 2013;19:35-42

4. Zhao G, Zhou X, Chen S, Miao H, Fan H, Wang Z, Hu Y, Hou Y. Differential expression of microRNAs in decidua-derived mesenchymal stem cells from patients with preeclampsia. J Biomed Sci 2014;21:81

5. Cutler AJ, Limbani V, Girdlestone J, Navarrete CV. Umbilical cord-derived mesenchymal stromal cells modulate monocyte function to suppress $\mathrm{T}$ cell proliferation. J Immunol 2010;185:6617-6623

6. Yen BL, Huang HI, Chien CC, Jui HY, Ko BS, Yao M, Shun CT, Yen ML, Lee MC, Chen YC. Isolation of multipotent cells from human term placenta. Stem Cells 2005;23: 3-9

7. Berkane N, Liere P, Oudinet JP, Hertig A, Lefêvre G, Pluchino N, Schumacher M, Chabbert-Buffet N. From pregnancy to preeclampsia: a key role for estrogens. Endocr Rev 2017;38: 123-144

8. Wan J, Hu Z, Zeng K, Yin Y, Zhao M, Chen M, Chen Q. The reduction in circulating levels of estrogen and progesterone in women with preeclampsia. Pregnancy Hypertens 2018;11:18-25

9. Jobe SO, Tyler CT, Magness RR. Aberrant synthesis, metabolism, and plasma accumulation of circulating estrogens and estrogen metabolites in preeclampsia implications for vascular dysfunction. Hypertension 2013;61:480-487

10. Li X, Song Y, Liu D, Zhao J, Xu J, Ren J, Hu Y, Wang Z, Hou Y, Zhao G. MiR-495 promotes senescence of mesenchymal stem cells by targeting Bmi-1. Cell Physiol Biochem 2017;42:780-796

11. Li X, Song Y, Liu F, Liu D, Miao H, Ren J, Xu J, Ding L, Hu Y, Wang Z, Hou Y, Zhao G. Long non-coding RNA MALAT1 promotes proliferation, angiogenesis, and immunosuppressive properties of mesenchymal stem cells by inducing VEGF and IDO. J Cell Biochem 2017;118:2780-2791

12. Liu L, Zhao G, Fan H, Zhao X, Li P, Wang Z, Hu Y, Hou Y. Mesenchymal stem cells ameliorate Th1-induced pre-eclampsia-like symptoms in mice via the suppression of TNF- $\alpha$ expression. PLoS One 2014;9:e88036

13. Zhao G, Miao H, Li X, Chen S, Hu Y, Wang Z, Hou Y. TGF- $\beta 3$-induced miR-494 inhibits macrophage polarization via suppressing PGE2 secretion in mesenchymal stem cells. FEBS Lett 2016;590:1602-1613

14. Smyth GK, Ritchie M, Thorne N, Wettenhall J. Limma: linear models for microarray data. In: Gentleman R, Carey V, Huber W, editor. Bioinformatics and Computational Biology Solutions Using R and Bioconductor: Statistics for Biology and Health. New York: Springer; 2005.

15. Tang Y, Li M, Wang J, Pan Y, Wu FX. CytoNCA: a cytoscape plugin for centrality analysis and evaluation of protein interaction networks. Biosystems 2015;127:67-72
16. Opsahl T, Agneessens F, Skvoretz J. Node centrality in weighted networks: generalizing degree and shortest paths. Soc Netw 2010;32:245-251

17. Cukierski WJ, Foran DJ. Using betweenness centrality to identify manifold shortcuts. Proc IEEE Int Conf Data Min 2008;2008:949-958

18. Du Y, Gao C, Chen X, Hu Y, Sadiq R, Deng Y. A new closeness centrality measure via effective distance in complex networks. Chaos: Interdiscip J Nonlinear Sci 2015;25: 033112

19. Bader GD, Hogue CW. An automated method for finding molecular complexes in large protein interaction networks. BMC Bioinformatics 2003;4:2

20. Yu Q, Peng C, Ye Z, Tang Z, Li S, Xiao L, Liu S, Yang Y, Zhao M, Zhang Y, Lin H. An estradiol-17 $\beta$ /miRNA-26a/ cyp19ala regulatory feedback loop in the protogynous hermaphroditic fish, Epinephelus coioides. Mol Cell Endocrinol 2019. [Epub ahead of print]

21. Pérez-Cremades D, Mompeón A, Vidal-Gómez X, Hermenegildo C, Novella S. miRNA as a new regulatory mechanism of estrogen vascular action. Int J Mol Sci 2018;19:E473

22. Liu G, Lu Y, Mai Z, Liu R, Peng Z, Chen L, Chen Z, Wang R, Ai H. Suppressing MicroRNA-30b by estrogen promotes osteogenesis in bone marrow mesenchymal stem cells. Stem Cells Int 2019;2019:7547506

23. Sethna F, Feng W, Ding Q, Robison AJ, Feng Y, Wang H. Enhanced expression of ADCY1 underlies aberrant neuronal signalling and behaviour in a syndromic autism model. Nat Commun 2017;8:14359

24. Shen JX, Cooper DM. AKAP79, PKC, PKA and PDE4 participate in a Gq-linked muscarinic receptor and adenylate cyclase 2 cAMP signalling complex. Biochem J 2013;455:4756

25. Gether U. Uncovering molecular mechanisms involved in activation of G protein-coupled receptors. Endocr Rev 2000; 21:90-113

26. Jekabsons K, Riekstina U, Parfejevs V, Laizane A, Pavasare M, Lencberga N, Jansone B, Muceniece R. Culture-expanded human dermal stem cells exhibit donor to donor differences in cAMP generation. Cell Tissue Res 2011;345:253-263

27. Drucker DJ. Glucagon-like peptides: regulators of cell proliferation, differentiation, and apoptosis. Mol Endocrinol 2003;17:161-171

28. Yang DC, Tsay HJ, Lin SY, Chiou SH, Li MJ, Chang TJ, Hung SC. cAMP/PKA regulates osteogenesis, adipogenesis and ratio of RANKL/OPG mRNA expression in mesenchymal stem cells by suppressing leptin. PLoS One 2008;3: e1540

29. Siddappa R, Martens A, Doorn J, Leusink A, Olivo C, Licht R, van Rijn L, Gaspar C, Fodde R, Janssen F, van Blitterswijk C, de Boer J. cAMP/PKA pathway activation in human mesenchymal stem cells in vitro results in robust bone formation in vivo. Proc Natl Acad Sci U S A 2008; 105:7281-7286

30. Lu LL, Liu YJ, Yang SG, Zhao QJ, Wang X, Gong W, Han ZB, Xu ZS, Lu YX, Liu D, Chen ZZ, Han ZC. Isolation 
and characterization of human umbilical cord mesenchymal stem cells with hematopoiesis-supportive function and other potentials. Haematologica 2006;91:1017-1026

31. Yang A, Zhang H, Sun Y, Wang Y, Yang X, Yang X, Zhang H, Guo W, Zhu G, Tian J, Jia Y, Jiang Y. Modulation of FABP4 hypomethylation by DNMT1 and its inverse interaction with miR-148a/152 in the placenta of preeclamptic rats and HTR-8 cells. Placenta 2016;46:49-62

32. Zhang L, Yuan JM, Zhao RH, Wang LM, Tu ZB. Correlation of MiR-152 expression with VEGF expression in placental tissue of preeclampsia rat and its influence on apoptosis of trophoblast cells. Eur Rev Med Pharmacol Sci 2019; 23:3553-3560

33. Li Q, Long A, Jiang L, Cai L, Xie LI, Gu J, Chen X, Tan L. Quantification of preeclampsia-related microRNAs in maternal serum. Biomed Rep 2015;3:792-796

34. Shi C, Zhang $M$, Tong $M$, Yang L, Pang L, Chen L, Xu G, Chi X, Hong Q, Ni Y, Ji C, Guo X. miR-148a is associated with obesity and modulates adipocyte differentiation of mesenchymal stem cells through Wnt signaling. Sci Rep
2015;5:9930

35. Jacenik D, Cygankiewicz AI, Fichna J, Mokrowiecka A, Małecka-Panas E, Krajewska WM. Estrogen signaling deregulation related with local immune response modulation in irritable bowel syndrome. Mol Cell Endocrinol 2018;471: 89-96

36. He SZ, Li J, Bao HC, Wang MM, Wang XR, Huang X, Li FH, Zhang W, Xu AL, Fang HC, Sheng YX. G protein-coupled estrogen receptor/miR-148a/human leukocyte antigen-G signaling pathway mediates cell apoptosis of ovarian endometriosis. Mol Med Rep 2018;18:1141-1148

37. Rao ZZ, Zhang XW, Ding YL, Yang MY. miR-148a-mediated estrogen-induced cholestasis in intrahepatic cholestasis of pregnancy: Role of PXR/MRP3. PLoS One 2017;12: e0178702

38. Chen MJ, Cheng YM, Chen CC, Chen YC, Shen CJ. MiR148a and miR-152 reduce tamoxifen resistance in ER+ breast cancer via downregulating ALCAM. Biochem Biophys Res Commun 2017;483:840-846 